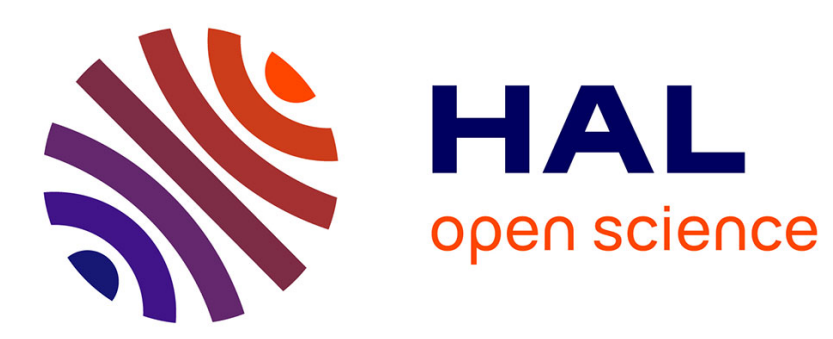

\title{
Geographic Routing Protocol for Peer-to-Peer Smart Grid Neighborhood Area Network
}

\author{
Mouna Rekik, Zied Chtourou, Nathalie Mitton
}

\section{To cite this version:}

Mouna Rekik, Zied Chtourou, Nathalie Mitton. Geographic Routing Protocol for Peer-to-Peer Smart Grid Neighborhood Area Network . The 15 IEEE International Conference on Environment and Electrical Engineering, Jun 2015, Rome, Italy. hal-01139120v2

\section{HAL Id: hal-01139120 \\ https://hal.inria.fr/hal-01139120v2}

Submitted on 18 Jul 2015

HAL is a multi-disciplinary open access archive for the deposit and dissemination of scientific research documents, whether they are published or not. The documents may come from teaching and research institutions in France or abroad, or from public or private research centers.
L'archive ouverte pluridisciplinaire HAL, est destinée au dépôt et à la diffusion de documents scientifiques de niveau recherche, publiés ou non, émanant des établissements d'enseignement et de recherche français ou étrangers, des laboratoires publics ou privés. 


\section{Geographic Routing Protocol for Peer-to-Peer Smart Grid Neighborhood Area Network}

\author{
Mouna Rekik \\ Inria Lille - Nord Europe, France \\ CMERP, Sfax, Tunisia. \\ Email: mouna.rekik@inria.fr
}

\author{
Zied Chtourou \\ CMERP, Sfax, Tunisia. \\ Email: ziedchtourou@cmerp.net
}

\author{
Nathalie Mitton \\ Inria Lille - Nord Europe, France. \\ Email: nathalie.mitton@inria.fr
}

\begin{abstract}
The distribution segment of the power system is of great importance in the smart grid (SG). The deployment of Information and Communication Technologies (ICTs) to support conventional grid will solve legacy problems that used to prevent implementation of smart services such as smart metering, Demand Side Management (DSM) or the integration of Distributed Energy Resources (DERs) within the SG. In this contribution, GRACO, a new geographic routing algorithm is proposed to support exploitation of SG full potential. We demonstrate, through simulations, the effectiveness of GRACO in terms of scalability, peer-to-peer routing, end-to-end delay and delivery rate.
\end{abstract}

Keywords-Smart Grid - Neighborhood Area Network - Wireless Sensor Network - Peer-To-Peer communication - routing protocol - geographic routing

\begin{tabular}{ll} 
& \multicolumn{1}{c}{ Acronyms and Abbreviations } \\
\hline SG & Smart Grid \\
DER & Distributed Energy Ressource \\
ICT & Information and Communication Technology \\
SGCN & Smart Grid communication network \\
HAN & Home Area Network \\
NAN & Neighborhood Area Network \\
WAN & Wide Area Network \\
AMI & Advanced Metering Infrastructure \\
FAN & Field area network \\
DSM & Demand-side management \\
DR & Demand Response \\
DA & Distribution Automation \\
RPL & Routing Power for Low power and Lossy network \\
GPSR & Greedy Perimeter Stateless Routing \\
DAP & Data Aggregation Point \\
ACO & Ant Colony Optimization \\
GRACO & geographic GReedy routing with ACO based recovery \\
P2MP & Point-to-Multipoint \\
MP2P & Multipoint-to-Point \\
P2P & Peer-To-Peer \\
IED & Intelligent Electric device \\
IETF & Internet Engineering Task Force \\
LLN & Low power and Lossy Network \\
ROLL & Routing Over Low power and Lossy networks \\
DODAG & Destination Oriented Directed Acyclic Graph \\
DAG & Directed Acyclic Graph \\
GR & Geographic routing \\
&
\end{tabular}

\section{INTRODUCTION}

The Smart Grid (SG) is the expansion of the traditional electrical grid with the integration of Distributed Energy Resources (DERs) and Information and Communication Technologies (ICTs), to better meet the increasing energy demand and environmental regulations.

The communication infrastructure is one of the key elements to enable smart grid applications. SG communication network (SGCN) is divided into 3 networks based on the coverage range and data rate: Customer premises area network or Home Area
Network (HAN), Neighborhood Area Networks (NAN) and Wide Area Network (WAN).

NAN ensures communication in the distribution grid. It is composed of Advanced Metering Infrastructure (AMI) and Field area network (FAN). The AMI provides a two way smart meter-utility communication in order to collect, measure, and analyze energy consumption data for grid management, outage notification, and billing purposes [1]. Moreover, the smart meter could be used as a HAN gateway in order to implement Demand-side management (DSM). DSM is a service that aims to ensure stability on the electricity grid. In fact, instead of adding more expensive generation to the system, DSM tries to balance electricity supply and demand in real time. It consists of a set of programs and activities designed to allow and encourage consumer to modify their electricity usage habits in order to reduce their consumption during peak periods. DSM comprise demand response (DR) programs and energy efficiency and conservation programs [2]. DR, called also load shifting, consists of transferring customer load during period of high demand to off-peak periods. Energy conservation programs encourage customers to give up some energy use in return for saving money, for example, turning up the thermostat a few degrees in summer to reduce air conditioning. On the other hand, Energy efficiency programs, such as replacing old electrical device with a more energy efficient one, allow customers to use less energy while receiving the same level of end-service and comfort.

On the other hand, the FAN ensures communication between field area devices and the control center. It enables a set of applications in the distribution level such as advanced Distribution Automation (DA). DA allows utilities to remotely monitor and control assets in its distribution network through automated decision-making, providing more effective fault detection and power restoration [1]. Besides, FAN enables one of the most important promises of the SG. Namely, better and more uniform integration of DERs into the grid, in particular renewable energy sources for a sustainable energy system with less environmental problems, more diversified energy resources and enhanced energy efficiency [1].

In this paper, the NAN is modeled as large scale network composed of a big number of wireless nodes (meters, sensors, field area devices, gateway). The routing protocol is a key factor for the performance of the SGCN. [3] reviews routing protocols for the SG NAN. Geographic routing and RPL emerge as the most promising candidates for routing traffic within the SG NAN. [4] compared RPL and GPSR for the case of NAN cluster with a unique aggregation point and 1000 smart 
meters with uniform random placement and a node density of 2000 nodes $/ \mathrm{km} 2$. They concluded better performance of the RPL in terms of delivery rate and end to end delay for packets sent from the smart meters to the DAP. They also showed that smart meters far from the DAP are responsible for delivery rate deterioration for the GPSR.

In this contribution, we are interested in more general peer to peer traffic routing scheme. We propose a new geographic routing protocol that ameliorates GPSR for communication in SG NAN.

In the remainder of the paper, we start in section II by providing arguments for peer to peer communications in $\mathrm{SG}$ NAN. Than, in section III we overview RPL routing and show that it's not suitable for peer to peer communications. The GRACO algorithm is presented in section IV. Section $\mathrm{V}$ presents simulation results for different NAN densities and finally Section VI concludes the paper.

\section{COMMUNICATION SCHEMES FOR NAN}

There are essentially three schemes for data traffic in a communication network: Point-to-Multipoint (P2MP), Multipoint-to-Point (MP2P) and Peer-To-Peer (P2P). In P2MP and MP2P networks, the communication is based on a master/slave relationship, in fact, in P2MP, one source node can transmit packets to many nodes, and in MP2P, one destination node can receive information transmitted by many nodes. In the P2P scheme, it is not necessary that all the nodes be connected to the central node. Every device in the network is able to directly communicate with any other device without a need to pass by a central control.

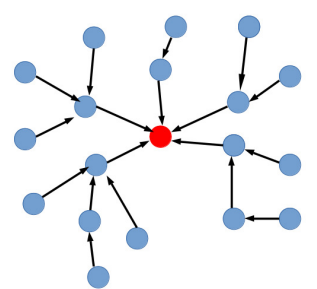

(a) MP2P

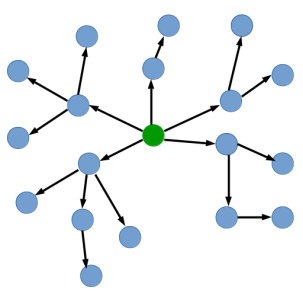

(b) P2MP

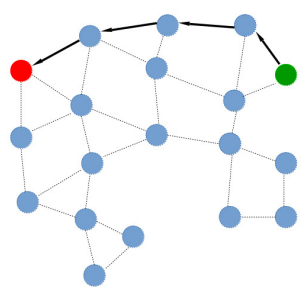

(c) P2P

Fig. 1: communication schemes

P2P networking has been investigated by the research community as a viable and effective approach to ensure scalability, self-organization and resiliency to the telecommunication infrastructure of SGs [5]. Many concepts related to SG can use any of the aforementioned traffic schemes. This is the case for the AMI where a great number of smart meters communicate with the an aggregation point within the distribution station to receive power reading signals (P2MP) and send their power readings (MP2P). However, some concepts, such as DERs integration, require $\mathrm{P} 2 \mathrm{P}$ communication. In fact, large scale integration of DERs can't be performed through conventional centralized control, it requires innovative distributed or agent based control strategies. In distributed controlled SG, the agents are intelligent electronic devices (IED), distributed all over the electric grid, and each is responsible for the control or measuring of a specific physical system. Typically, these IEDs are sensor nodes with some processing and memory capacity that are interconnected via an heterogeneous P2P communication network with stringent delay requirements on message delivery. On the other hand, the power grid is a delay-sensitive system, it has strict delay requirements since a delayed message can cause potential system failures. For example, a fault event message need to be delivered within a delay as low as $3 \mathrm{~ms}$, in order to isolate the affected segment, otherwise the whole system will be damaged. [6]

Hence, due to its high-reliability and time-critical requirements, $\mathrm{P} 2 \mathrm{P}$ communication scheme is a suitable networking solution for SG applications. In such system, devices will communicate with each other without a central monitoring using P2P scheme. A forwarded data packet will use a shorter path than the one used in a central control based topology. Besides, flooding one central node with huge traffic will increase significantly transmission delay and may also affect the reliability of the network. For these reasons, the P2P communication in distributed control systems is expected to reduce the transmission delay.

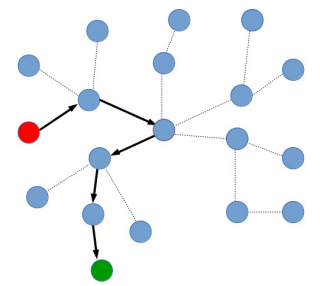

(a) P2P communication in center based topology

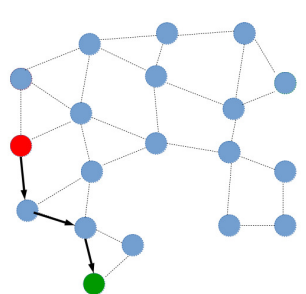

(b) P2P communication
Fig. 2: example

\section{Routing Protocol For Low Power AND Lossy NETWORKS (RPL)}

RPL [7] is a routing protocol for Low power and Lossy Networks (LLNs) designed by the IETF Routing Over Low power and Lossy networks (ROLL) Working Group.

The basic idea behind the RPL consists in constructing Direction Oriented Directed Acyclic Graphs (DODAG). These are are tree like structures with a root and one or many leafs, and in which a path is built for every leaf node to reach a unique root node. Each node is assigned a rank depending on an objective function and starting with the lowest rank at the root. The simplest objective function would use the number of hops between a node and its root. The DODAG construction starts by assigning rank 1 to the root. Than any node with direct connection to the root is assigned rank 2. Than any node that can see only rank 2 nodes is assigned rank 3 , and so 
on. Communications are directed from highest rank to lowest one.

A root starts the DODAG creation by sending DIO (DAG Information Object packet). A DIO message contains information about the DODAG such as the DAGID used to identify the DAG, rank information and the objective function.

When a node receives a DIO message and decides to join the DODAG, it should add the DIO sender to its parent list, compute its own rank, associated with this specific parent node using the corresponding objective function. And, finally broadcast the DIO message with the updated rank information. If a node loses connectivity, it sends out a DAG Information Solicitation (DIS) message and the neighboring nodes that hear it will respond with a DIO message announcing they are a part of a DODAG.

Using the constructed DODAG, each client node will be able to forward any upward traffic through its parent as the nexthop node.

To create downward routes from the root to a leaf node, the leaf node should issue a control message called Destination Advertisement Object (DAO). A DAO packet includes the rank information used by nodes to determine how far away is the destination. The DAO message will be forwarded to the root using the upward path indicated by the DODAG, and all the intermediate nodes record the reverse path information from the DAO message, after that, a complete downward path is established from the root to the leaf node.

The RPL instance defines DODAG networks that build their trees using the same objective function.

The RPL is optimized for P2MP and P2MP communication schemes [8]. For that reason, it was proposed as a routing solution for AMI networks [9][10] where traffic is limited from meters to a concentrator, and from a concentrator to meters. RPL does not provide an optimized P2P traffic support [10]. When a node sends a packet to another node, the packet uses upward route until arriving to a common ancestor at which point it is forwarded in the downward direction to the destination. For these reasons, RPL is not suitable for $\mathrm{P} 2 \mathrm{P}$ NAN. In the next session, we will propose the use of geographic routing for P2P NAN.

\section{Geographic GReedy routing with ACO BASED RECOVERY (GRACO)}

\section{A. Geographic routing for large scale P2P NAN}

Geographic routing (GR) is a class of routing approaches that exploit geographic position in routing decision in stead of topological information. It has been widely used in ad-hoc and wireless sensor networks for its diverse advantages. In fact, GR is a localized routing scheme since packet forwarding decision is achieved by using only information about the position of nodes in the vicinity and the position of the destination node, which minimizes significantly communication overhead. Hence, no additional information has to be stored neither on the nodes on the path, nor in the message which makes GR a memory-less and scalable routing [11]. Besides, GR is also distributed, every node performs the same algorithm [11]. As previously discussed, the deployment of a wireless communication network for the NAN faces many challenges. First of all, NAN is supposed to support different applications and multiple traffic flows, the communication network should then manage a huge amount of data, thus, the routing solution should deliver the data packets with the minimum overhead possible so it does not affect the network performances. As NAN is a large scale network with highly dynamic topology due to the often expansions to cover larger areas and additional endpoints, it needs a scalable routing solution. Besides, the communication scheme for NAN should be resilient to outdoors harsh communication environment and instability of wireless links. For these reasons, the simplicity, localized and distributed operation and the low overhead, memory-less and scalability features makes GR a very good candidate for NAN. Some research papers investigated in the application of geographic routing for AMI networks. Perimeter Stateless Routing (GPSR) [12] was proposed in this context. [13] studies of the feasability and the performance of GPSR in NAN scenarios. It was proved that GPSR can support different NAN services in terms of latency and reliability. The authors of [4] compared the performances of GPSR to RPL in the case of NAN network. The simulation results proved that RPL outperforms GPSR in terms of transmission reliability and delay.

\section{B. Overview of GRACO}

Geographic GReedy routing with ACO recovery strategy (GRACO) [14] combines a modified greedy forwarding and a recovery strategy based on swarm intelligence. In the beginning, GRACO makes the routing decision using geographic greedy forwarding strategy [11]. As the simplest form of geographic routing, greedy geographic routing inherits all its advantages. Using 1-hop location information, the source and each intermediate node select its closest neighbor to the destination as the next-hop node, thus packets are greedily forwarded in order to decrease their distance to the destination until arriving to the destination.

Geographic greedy forwarding mechanism suffers from the void problem, whenever a packet arrives to a node that can't find a closer node than itself to the destination, it can't progress and the node is a stuck node. For that reason, greedy forwarding is usually coupled with a recovery strategy. Indeed, when routing process gets stuck at an intermediate node, it will launch a recovery strategy. In this context, GRACO proposes an ACO based strategy to recover from the void problem. During recovery, two types of ant like packets are used: Fants to search for a path around the void and Bants to mark the paths found for later use. The Fants will be guided to the direction of the destination using zone concept. Once a Fant arrives to a node closer to the destination than the stuck node, a Bant is sent to mark the path found by dropping pheromone trails. The dropped pheromone trails will guide next data packets sent to the same destination. A data packet sent to a given destination will be forwarded using pheromone trails for this destination if exist, otherwise, the next hop is selected using plain greedy forwarding strategy. Thus, the first mode is a modified greedy routing that accounts for the pheromone trails. Simulations proved that GRACO avoids holes and produces near optimal paths. The performance of GRACO was compared to the greedy-face-greedy (GFG) routing algorithm [15]. Simulations results presented in [14], show that GRACO outperforms GFG in term of end-to-end delay, data delivery cost and hop count. Besides, GRACO provides a high data delivery rate. 


\section{Simulations AND Results}

The performance of GRACO is measured using the WSNET simulator [16].

We generate random topologies with different densities. We evaluate the algorithm according to end-to-end delay, delivery rate and hop count. The simulation results of GRACO are given with $95 \%$ confidence intervals. In order to simulate the scalability of the protocol, we run simulations on a network with increasing density. We choose to incrementally add nodes to the network in order to simulate the evolution of the NAN from early stages where just a few customers are enrolled in the network and the nodes are sparse across the selected region to more advanced stages with denser aggregation of nodes representing a more extended version of the NAN.

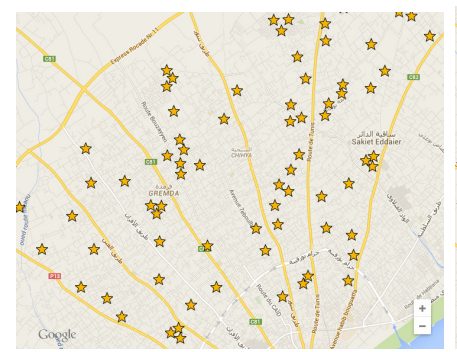

(a)

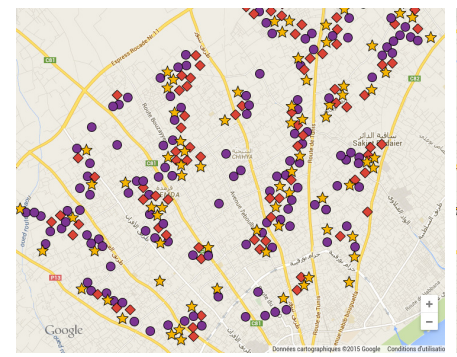

(c)

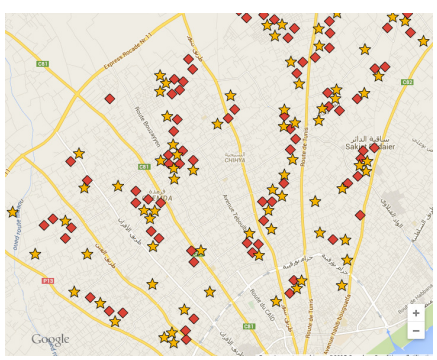

(b)

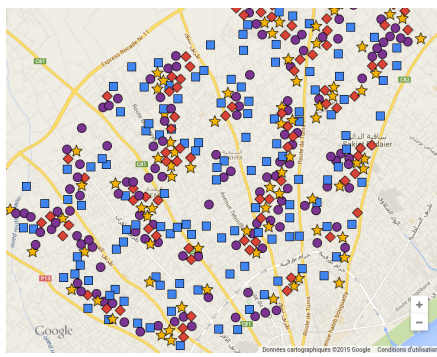

(d)
Fig. 3: Stages of NAN extension within an urban region

To vary the nodes degree, we vary their range from $25 \mathrm{~m}$ to $50 \mathrm{~m}$ by steps of $5 \mathrm{~m}$. Each combination of topology and algorithm is run 50 times. Error bars on curves symbolize a $95 \%$ confidence interval.

\begin{tabular}{ll} 
Parameter & Value \\
\hline Duration (s) & 300 \\
MAC layer & idealmac and 802.15.4 \\
Interferences & none \\
Density & $10,15,20,25,30,40$
\end{tabular}

TABLE I: Simulation parameters

In order to measure the impact of void zones on the algorithm performances, we choose a set of random sources and destinations where there is, necessary, a void to be handled in the routing process. One data packet is sent between a pair of source and destination each 10 s.

The performance of the routing protocol is measured in terms of the average route length, the end-to-end delay and the data delivery ratio. Table I summarizes the simulation parameters.
The simulation results show that the more the network is dense the better performs the proposed routing protocol.

The route length is the number of hops a data message needs to go from the source to destination. As the density of the network is increased, shorter paths are found. Specifically, as plotted in figure 4, the average route length drops from 59 hops at network density 10 down to 11.94 hops at network density 40 for an ideal mac layer, and from 37 hops at network density 10 down to 16.32 hops at network density 40 for 802.15 .4 mac layer.

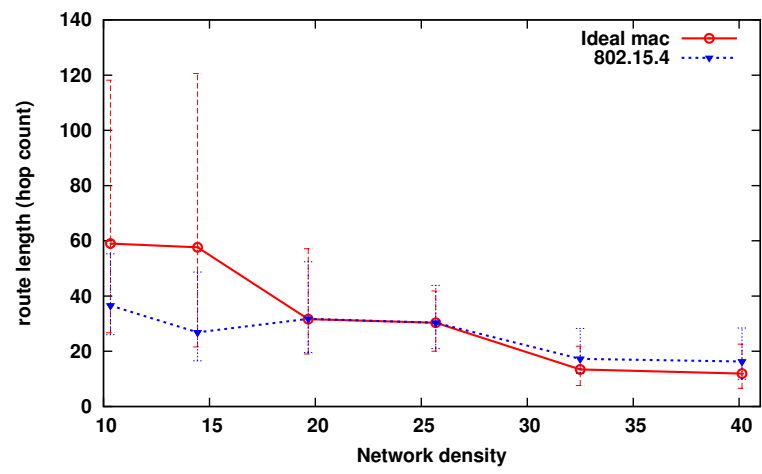

Fig. 4: hop-count

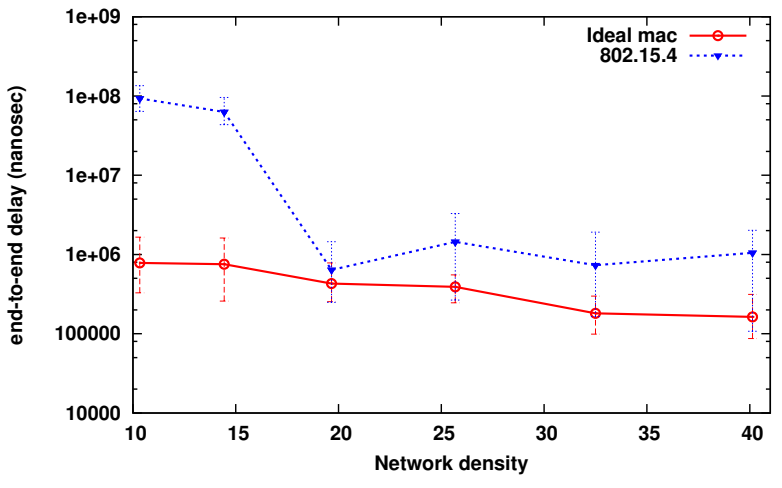

Fig. 5: end-to-end delay

Figure 5 shows the end-to-end delay varying with the network density. The end-to-end delay is the time interval between a given source sends a packet and the destination receives it. For an idal mac layer, the average end-to-end delay reduces from $0.781 \mathrm{~ms}$ at a network density 10 to $0.163 \mathrm{~ms}$ at a network density 40, and for 802.15.4 mac layer, it reduces from 93,183 $\mathrm{ms}$ at a network density 10 to $1,049 \mathrm{~ms}$ at a network density 40 .

The delivery ratio is the ratio of data packets successfully received by their destinations to all data packets sent by the sources. The data delivery rate is also improved when increasing network density. In fact, figure 6 shows that GRACO provides a $100 \%$ delivery rate with an ideal mac, however, for 802.15 .4 mac, a high reliability of packet transmission is achieved only in a high density network. 


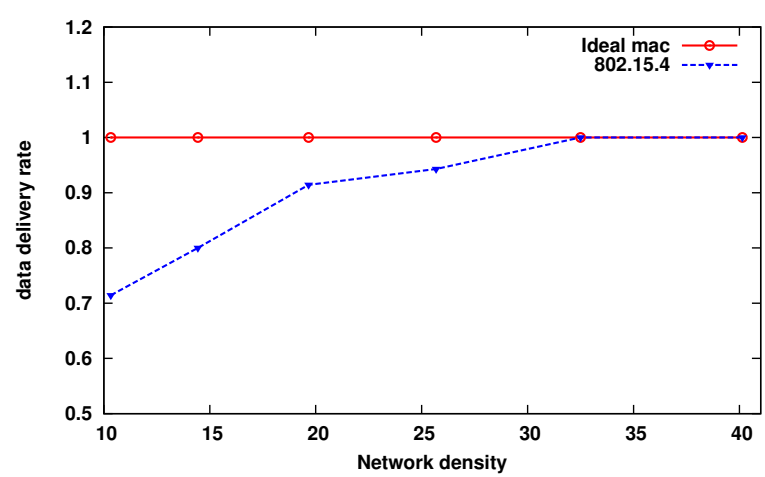

Fig. 6: Data delivery rate

\section{CONCLUSION}

In the paper, we proposed the use of geographic routing for NAN communication. The proposed routing protocol GRACO is an ACO aided geographic greedy forwarding that ensures P2P communication. Simulation results demonstrate that GRACO is scalable. Indeed, end-to-end delay, path length and delivery rate are improved with increasing network density or NAN size. This is consistent with the constant expansion of the network and the increasing need for larger communication traffic. The performance of GRACO shows that it is suitable for NAN communication. Delivery rate and end-to-end delay are within the NAN communication requirements.

\section{ACKNOWLEDGMENTS}

This work has been partially supported by a grant for the CPER CIA and the MESRST TIC.

\section{REFERENCES}

[1] U.S. Energy Department. Communications requirements of smart grid technologies. Technical report, Energy Department, 2010.

[2] B. Davito, H. Tai, and R. Uhlaner. The smart grid and the promise of demand-side management. McKinsey \& Company, 2010.

[3] N. Saputro, K. Akkayaa, and S. Uludagb. A survey of routing protocols for smart grid communications. Computer Networks, 56(11):2742 - 2771, 2012.

[4] Q. Ho, Y. Gao, G. Rajalingham, and T. Le-Ngoc. Performance and applicability of candidate routing protocols for smart grid's wireless mesh neighbor area networks. In Communications (ICC), 2014 IEEE International Conference on, pages 3682-3687, June 2014.

[5] C. Rottondi, G. Savi, M.and Verticale, and Ch. Krauß. Mitigation of $p 2 p$ overlay attacks in the automatic metering infrastructure of smart grids. 2012.

[6] Mingkui Wei and Wenye Wang. Toward distributed intelligent: A case study of peer to peer communication in smart grid. In Global Communications Conference (GLOBECOM), 2013 IEEE, pages 2210-2216. IEEE, 2013.

[7] Rpl: Ipv6 routing protocol for low power and lossy networks. http://tools.ietf.org/id/draft-ietf-roll-rpl-19.html, March 2011.
[8] G. Tuna, V. C. Gungor, and K. Gulez. Wireless sensor networks for smart grid applications: a case study on link reliability and node lifetime evaluations in power distribution systems. International Journal of Distributed Sensor Networks, 2013, 2013.

[9] D. Wang, Z. Tao, J. Zhang, and A.A. Abouzeid. Rpl based routing for advanced metering infrastructure in smart grid. In Communications Workshops (ICC), IEEE International Conference on, pages 1-6, May 2010.

[10] E. Ancillotti, R. Bruno, and M. Conti. The role of the rpl routing protocol for smart grid communications. Communications Magazine, IEEE, 51(1):75-83, January 2013.

[11] N. Mitton, T. Razafindralambo, and D. Simplot-Ryl. Position-based routing in wireless ad hoc and sensor networks. In Theoretical Aspects of Distributed Computing in Sensor Networks. Springer, 2010.

[12] B. Karp and H.-T. Kung. Gpsr: Greedy perimeter stateless routing for wireless networks. In Proceedings of the 6th annual international conference on Mobile computing and networking, pages 243-254. ACM, 2000.

[13] Q. Ho, G. Rajalingham, and T. Le-Ngoc. Performance and applicability of geographic-based routing in smart grid's neighbor area networks. In Advanced Technologies for Communications (ATC), 2013 International Conference on, pages 215-219. IEEE, 2013.

[14] M. Rekik, N. Mitton, and Z. Chtourou. Geographic greedy routing with aco recovery strategy graco. In $A d$ Hoc Now 2015, Athens, Greece, June 2015.

[15] P. Bose, P. Morin, I. Stojmenović, and Jorge Urrutia. Routing with guaranteed delivery in ad hoc wireless networks. In Proceedings of the 3rd International Workshop on Discrete Algorithms and Methods for Mobile Computing and Communications, DIALM '99, pages 4855, New York, NY, USA, 1999. ACM.

[16] Wsnet simulator. http://wsnet.gforge.inria.fr/. 